\title{
Modeling of the interface between the concrete and the fibers grid in concrete slab
}

\author{
Bouzeboudja F*, Ali Ahmed C
}

\footnotetext{
Laboratory LaMoMS/University Mouloud Mammeri, Tizi-Ouzou, Algeria,

* Corresponding Author: bouzeboudjaf@yahoo.fr
}

Received: 14-02-2018

Revised: 20-05-2018

Accepted: 23-05-2018

\begin{abstract}
Abstract. Fiber grid reinforcements are widely used in construction, especially in the rehabilitation of structures; short fibers are also used in concrete. However, reinforcing mortars with textiles (fiber gratings) which offers an improved concrete, and a wide variety of new and exciting architectural forms, is still a relatively new application that shows a need for research. Tests on the use of Glass Fiber Grids (GFG) and Polypropylene Grids (PPG) in the concrete matrix which can be an interesting solution to improve the bearing capacity and change the failure mode of slabs subjected to a load of punching have been realized. The results showed a significant increase in the bearing capacity, a better distribution of the cracks, as well as an improvement of the deflexion before failure; compared to unreinforced reference slabs.

In order to understand the behaviour of the concrete-GFG interface of the tested slabs, a modeling of this latter was carried out using a constitutive law expressing the shear stress between the two materials as a function of the relative sliding between the surfaces in contact, taking into account the mechanical characteristics of both materials. The analysis of the bond-slip curve obtained has shown that the use of the glass fiber grid inside the concrete matrix allows a better behaviour of the interface between constituent materials with respect to the glued fiber-reinforced polymer (FRP) plates on the surface of the slab.
\end{abstract}

Key words: Concrete slab, reinforcement, grids, punching, interface stress, slip, modeling.

\section{Introduction}

New techniques that involve the use of composite materials in construction are currently being studied in different works (Ait Tahar and Bouzeboudja, 2011). These methods are booming as these innovative materials have important advantages over traditional materials such as: lightness, mechanical and chemical resistance, freedom of shape and prolonged life.

The use of textile reinforced concrete (TRC) is a very effective method for strengthening reinforced concrete (RC) constructions. Vast research on TRC was carried out, so as to examine the use of TRC for subsequently strengthening the bending load capacity of existing concrete or reinforced concrete elements (Carozzi et al., 2016). FRP (fiber reinforced polymer) is a composite material used in the rehabilitation of structures such as the reinforcement of beams, slabs and columns (Neale et al., 2011).

High modulus fibers can be used to improve stiffness and crack resistance. In order to greatly improve the structural strength, the high strength fibers can be used in conjunction with fibers having ductile behaviour, which exhibit some strain hardening until the high-strength fibers break (Wang et al, 2010).The latter can be used to control the deformation of the elements of the structures. In addition, the service life can be increased by mixing high ductility fibers with high strength fibers.

In the framework of this work, we have proposed a method of internal reinforcement of concrete slabs, by GFG and PPG grids, embedded in the concrete matrix at the level of the lower fibers and 
top of the slab, to improve the bearing capacity and change the failure mode of these elements. This method consists of carrying out additional reinforcement to the traditional steel. Indeed; an experimental study was carried out on reinforced slabs of GFG, and PPG, subjected to a progressive centered punching load.

The bond between the fibers and the matrix has a considerable influence on this material behaviour and is very difficult to model because of the non-uniform stress distribution over a roving section (Soranakom and Mobasher, 2009). A study of the interfacial behaviour of the materials used for making the tested slabs, using an existing model in the literature to establish a relationship between the slip and the adhesion stress developed at the interface between the two materials is presented.

\section{Experimental program}

In order to demonstrate the advantage of reinforcement of concrete slabs with textiles against the loading of punching, the main objective of this experimental investigation consists in carrying out a characterization of the structural behaviour of bidirectional slabs armed with glass fibers and polypropylene, grids of large and small stitch.

An experimental investigation was carried out, using slabs measuring $280 \times 230$ x $70 \mathrm{~mm}$ armed with two grids (see fig 2). Two variants are considered: nine slabs reinforced by grids with small stitch and nine slabs reinforced by grids with large stitch. For each variant, a combination of grids is adopted to highlight the influence of the compatibility of the mechanical work of the grids, the influence of the position of the grid in the slab on the recovery of the applied load and on the mode of failure To ensure good reproducibility of the results, at least three specimens for each variant are tested. Three unreinforced concrete slabs, considered as reference slabs, are also produced for the comparison of the results.

To highlight the influence of grid mesh dimensions on the bearing capacity of the reinforced slab and especially on the failure mode, two types of mesh were considered in this study: small meshes and large meshes (Figure 1).

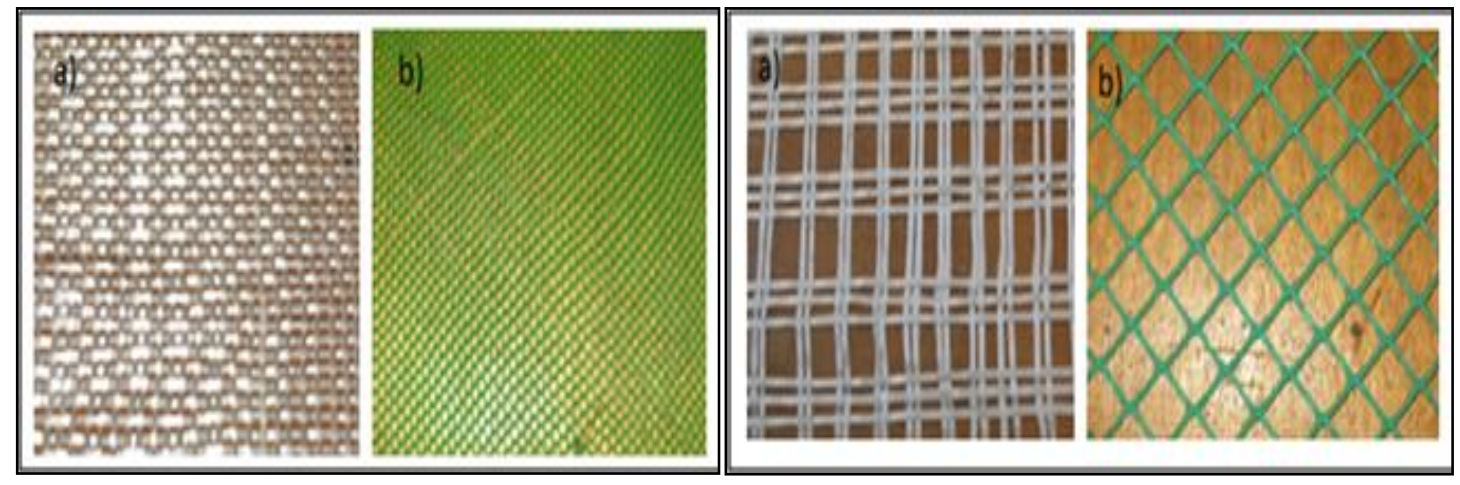

Fig 1. Grids of small meshes and larges meshes:

a) GPG

b) PPG

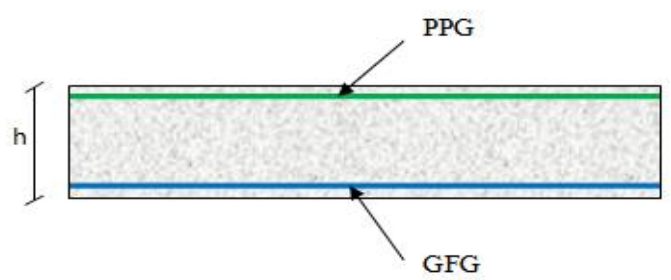

Fig 2. Disposition of the grids in the slab 


\subsection{Punching tests}

The slabs are tested under punching load (Fig 3), applied using a hydraulic cylinder, through a rigid metal plate with $30 \mathrm{~mm}$ sides and a thickness of $1 \mathrm{~cm}$. the slab is simply supported on a steel support device, consisting of four supposedly infinitely rigid sides. During the test, the value of the load and the vertical deflexion corresponding to the middle of the slab are read automatically.

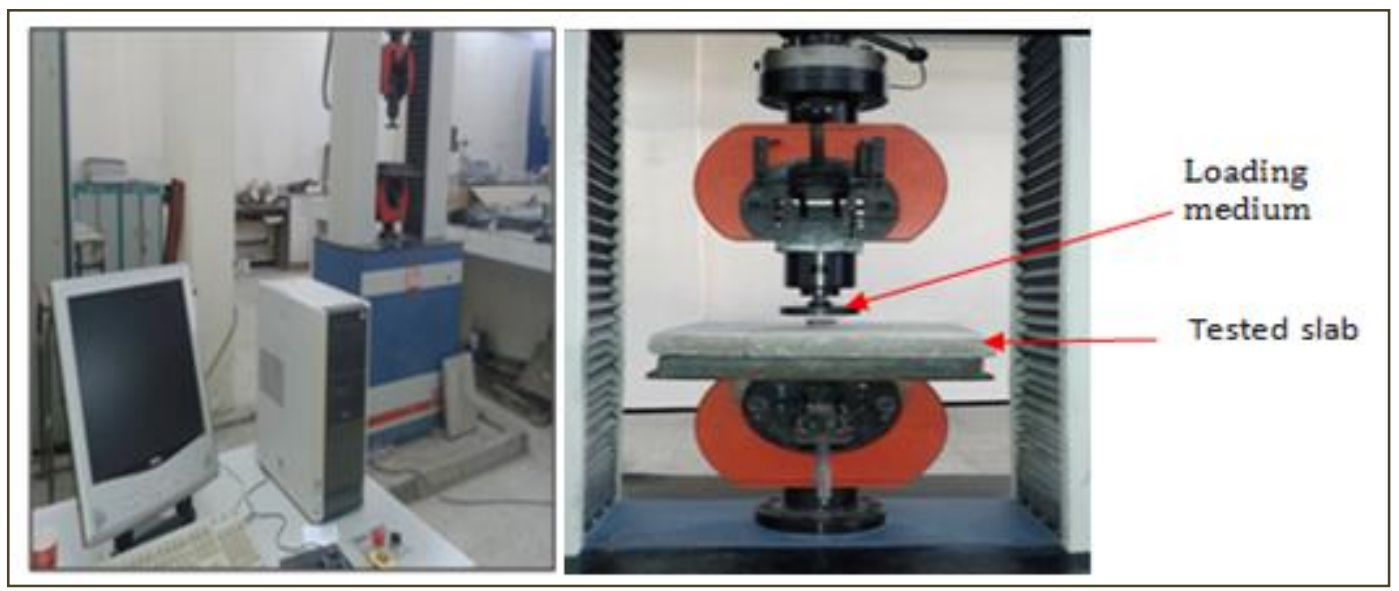

Fig 3. IBERTEST test machine and support device.

\subsection{Results and discussion}

On fig 4 are confronted the curves load -deflection corresponding, to the slabs tested.

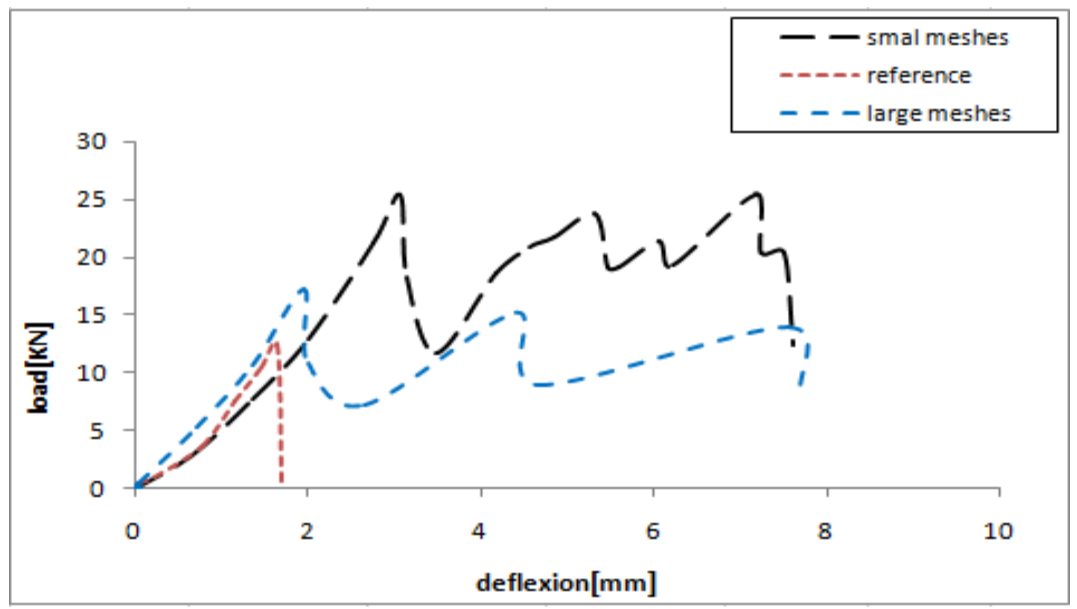

Fig 4. Confrontation of the 'load-deflection' curves of the slab reinforced by two GFG / GPP grids and the reference slab.

It can be seen that a considerable improvement in resistance and ductility is obtained with the reinforced slabs by the combination of GFG and PPG grids. Indeed, unreinforced slab exhibits a fragile behaviour with an ultimate tensile load equal to $12.45 \mathrm{kN}$ and a corresponding deflexion equal to $1.65 \mathrm{~mm}$, on the other hand the rupture of the reinforced slab is obtained under an ultimate load of $25.42 \mathrm{kN}$ and a corresponding deflexion of $3,07 \mathrm{~mm}$. This reinforcement of slabs has a gain in terms of bearing capacity of the order of $104.18 \%$ and of ductility estimated at $86.06 \%$.

The slabs reinforced with grids of small meshes have a better behaviour compared to the slabs armed with large meshes because the approximation of the mesh threads allows obtaining a 
homogeneous reinforcement of the slab and a better distribution of stresses around the most stretched fiber, as well as an increase in the percentage of reinforcement.

The various results obtained highlight the interest of the internal reinforcement of concrete slabs by GFG and PPG grids; in fact, the GFG grid considerably increases the ultimate load and allows a better distribution of cracks in the tension zone, while the PPG grid allows the development of the flexional mechanisms in the slab, which leads to a more ductile mode of rupture compared to the reference concrete slabs.

\section{Aspect characterizing the bending behaviour of slabs}

The different aspects characterizing the flexional behaviour of the slabs were observed on the slab reinforced by the combination of GFG and PPG grids. Indeed, during the loading, the behaviour of the reinforced slab passes through several successive phases, each one being characterized by a resistance to the peak and a corresponding deflexion. The first cracks create local discontinuities of the concrete matrix and a detachment of the concrete-GFG interface. When the different blocks are separated, the grids act as tie rods to secure and connect the blocks of the slab. When the PPG grid gives way, the slab is completely ruined. This behaviour is characterized by four phases as illustrated in Fig. 5.

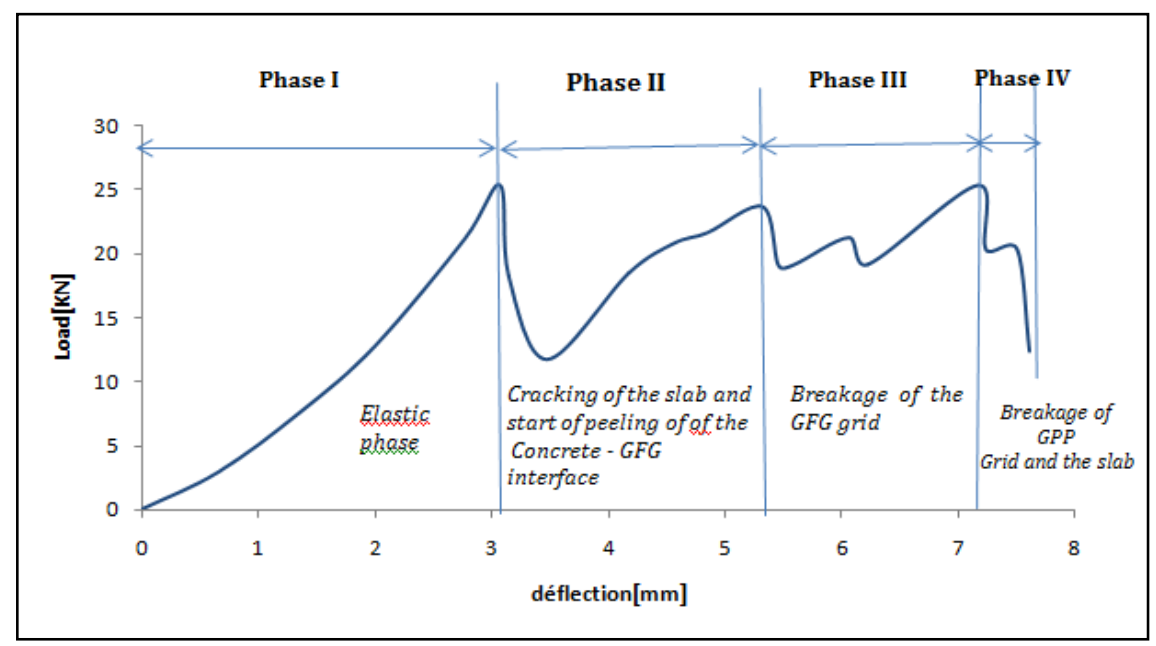

Fig 5. Phases of the behaviour of the slab reinforced by 'GFG / PPG' small meshes

> Phase I: This phase corresponds to the elastic behaviour of uncracked concrete, characterized by a rapid increase in load, up to $25.42 \mathrm{kN}$, corresponding to a deflexion of $3,07 \mathrm{~mm}$. The first peak corresponds to the elastic limit value. At the end of this phase, there is a rapid drop in resistance due to the initiation and propagation of the first cracks;

Phase II: During this phase, cracks appear on the tensile face of the slab, and the deflexion increases more rapidly, the fall of the load is due to the peeling at the concretegrid interface GFG on both sides of the crack. The grid GFG then plays the role of tie-rod to secure and connect the "cracked blocks" of the slab. There was then a resumption of resistance;

$>$ Phase III: In this phase, a drop in the charge occurs suddenly, which corresponds to the breakage of the GFG grid. Only the PPG grid continues to withstand the stresses developed by the applied load. This phase is very short because the PPG grid is weakly resistant to the applied load and deforms rapidly;

$>$ Phase IV: This phase corresponds to the breakage of all the components of the slab. 


\section{Behaviour of FRP composite materials}

Fig. 6 illustrates the idealized stress-strain curve of hybrid FRP composites composed of three types of high modulus, strength and ductility fibers.

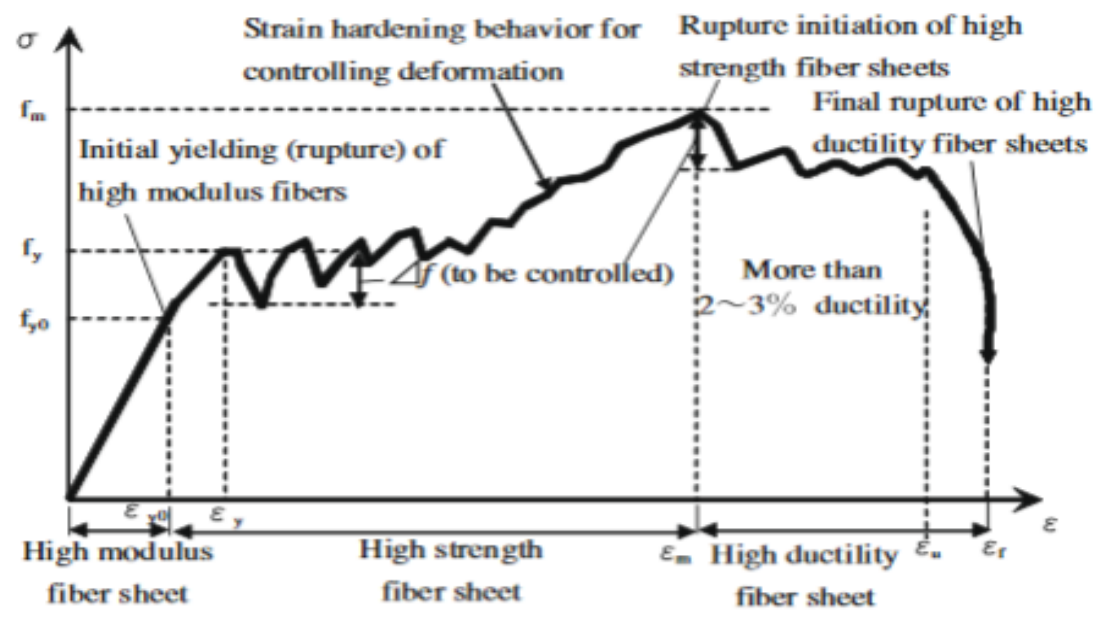

Fig. 6. Idealized strain-strain curve of hybrid PRF composites (Wang et al, 2010)

Theoretical studies have shown that stiffness can be improved by fibers of higher modulus and the ductile behaviour can be obtained by hybrid fibers (Wang et al, 2010). But the main problem often shown is the premature failure of the composites interface occurring due to the locally high stress concentration at the concrete-fiber interface.

Existing tear tests have shown that in the vast majority of cases and with the exception of cases where a very weak adhesive or a very strong concrete is used, the rupture of a FRP-concrete interface is obtained by the development of cracks in the concrete layer adjacent to the adhesive layer, in case where this plate is narrower than the concrete block (Neale et al, 2005) . During the load increase, the breaking plane propagates from the loaded end to the free end of the FRP plate.

A very important aspect of the behaviour of these bonded joints is the existence of an effective length of the adhesion beyond which an increase in the adhesion length $L$ cannot improve the ultimate load. This constitutes a fundamental difference between an externally glued plate and internal reinforcement for which a sufficient anchorage length can always be found in order to ensure complete tensile strength of the reinforcement.

Since the behaviour of FRP composites (Fig 6) is similar to that of the slabs tested in our study (Fig 4), an existing model characterizing the interface between the FRP plate and the concrete will be used to establish bond-slip relationship characterizing the interface between the concrete and the textile used.

\section{Interface Behaviour}

After the cracking of the concrete, the interface between the reinforcing fiber and the concrete has an important influence on the overall behaviour of the textile reinforced concrete. For that, several researchers are interested in the analytical modeling of the matrix fiber interface (Carozzi et al., 2016) , others with the numerical simulation of the behaviour of this composite material, while some are interested in testing the pull-out of the textile from the concrete matrix (Häußler-Combe and Hartig, 2007; Lorenz, 2012). For our proposed internal reinforcement of the slabs, an analytical model will be used to characterize the interface between the GFG grid and the concrete matrix. 


\subsection{Grid removal mechanism}

In order to develop a law of the flexible and unified material, the constitutive law of the interfacial bond, the reaction of the wire to the longitudinal tensile stress, and the stress at the junction of the longitudinal and transverse wires are described as segmental linear functions (see Fig. 7).

Figure 7.a shows a model of slip at the interface of the pre- and post- maximum shear stress $\tau$ as a function of the slip S. Figure 7.b presents a typical model of longitudinal tensile stress which could start with an initial release. Finally, a nonlinear spring model, as shown in Figure 7.c represents the additional stiffness gappearing at the junction (crossing) of the wires (Soranakom and Mobasher, 2008).

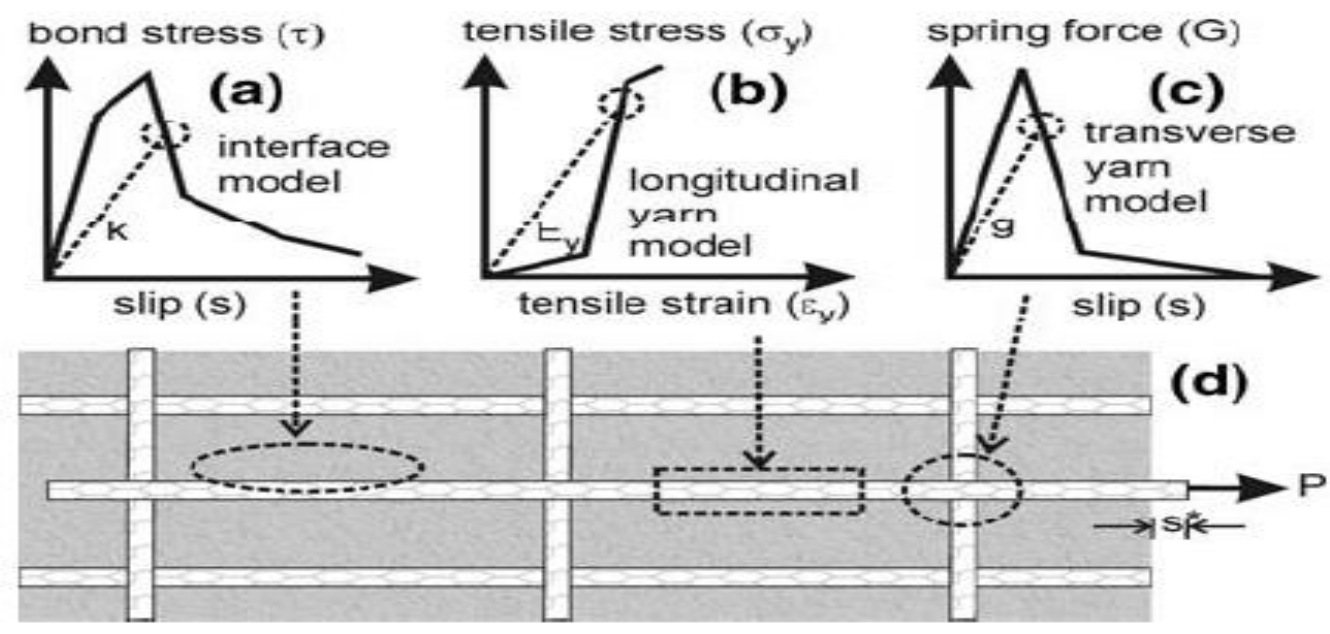

Fig.7. Scheme of the grid removal mechanism; (a) interface link slip model; (b) of the longitudinal wire pattern; (c) spring model simulating the anchoring force at the junction cross wire; (d) the composite sample (Soranakom and Mobasher , 2008)

With the use of multilinear segments to describe material behavior models and the use of intersection modules to control the behavior of the latter, the three models can be implemented as piecewise linear functions in the finite difference model.

\subsection{Stress - slip relationship}

Figure 8 illustrates the relationship between the tangential stress $\tau$ and the slip $\mathrm{S}$, this pace is the same for all interfaces., where $\tau(\mathrm{MPa})$ is the local bond (shear) stress, $S(\mathrm{~mm})$ is the local slip, $\tau \max (\mathrm{MPa})$ is the local bond strength (i.e. the maximum bond/shear stress experienced by the interface), $S_{0}(\mathrm{~mm})$ is the slip when the bond stress reaches $\tau_{\max }$ (Neale et al., 2005).

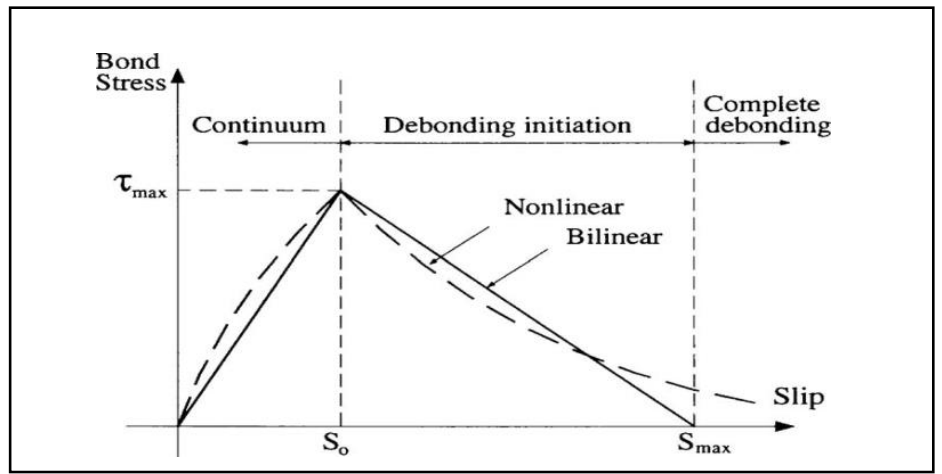

Fig. 8. Lay of the constitutive law constraining of bond-slip (Neale et al., 2005). 
It is composed of a first linear elastic part, followed by a second part which becomes progressively non-linear as the slip increases. Simultaneously, the plastic deformation in the interface increases to rupture. The post-rupture behaviour can be described by a reduction of the adhesion stress with the increase of the slip. Schematically, this relationship can be represented by bilinear behaviour (Fig 8), whose segments have the following characteristics:

- Segment 1: elastic linear behaviour, going from $S=0$ to $S=S_{0}$, with: $S_{0}$ being the slip corresponding to the end of the linear elastic domain, and to the maximum adhesion stress $\tau$ max.

- Segment 2: locally linear behaviour, representing the inelastic and softening discharge, for $\mathrm{s}>\mathrm{s}_{0}$.

\subsection{Model of Lu and Teng}

Since it is difficult to obtain accurate bond-slip curves directly from strain measurements in a pull out test, Lu et al. (2005) recently explored a numerical approach from which the bond-slip curve of any point along the interface can be obtained. The approach is based on the observation that debonding in a pull test occurs in the concrete, so if the failure of concrete can be accurately modelled, the interfacial shear stress and slip at a given location along the interface can be obtained from the finite element model.

A simplified model without significant loss of accuracy can be readily obtained by noting that the initial stiffness of the bond-slip curve is much greater than the secant stiffness at the maximum point. Based on this observation, the initial stiffness can be approximated as the infinite and the following simplified bond-slip model can be obtained by the relations (1a) and (1b) (Lu et al., 2005)

$$
\begin{gathered}
\tau=\tau_{\max } \frac{s}{S_{0}} \\
\tau=\tau_{\max } \mathrm{e}^{-\alpha\left(\frac{\mathrm{s}}{\mathrm{s}_{0}}-1\right)}
\end{gathered}
$$

The shear stress $\tau_{\max }$ and the corresponding slip $s_{0}$ are given by

$$
\begin{gathered}
\tau_{\text {max }}=\alpha \beta_{\omega} f_{t} \mathrm{~V} \\
S_{0}=0.0195 \beta_{\omega} f_{t}
\end{gathered}
$$

$\beta_{\mathrm{w}}$ be calculated with equations:

$\beta_{\omega}=\sqrt{\frac{2-{ }^{{ }^{b}} / b_{c}}{1+{ }^{b_{f}} / b_{c}}}$

$b_{c}$ width of concrete;

$b_{f}$ width of renfort;

The parameter $\alpha$ controls the shape of the descending branch of the curve, and is given by :

$$
\alpha=\frac{1}{\frac{G_{f}}{\tau_{\max S_{0}}}-\frac{2}{3}}
$$

The interfacial fracture energy $\left(\mathrm{G}_{\mathrm{f}}\right)$ can be expressed as : 
$G_{f}=0.308 \beta_{\omega}^{2} \sqrt{f_{t}}$

Where $f_{\mathrm{t}}$ is the tensile strength of concrete.

\subsection{Application of the simplified model of Lu and Teng to the case of slabs reinforced by grids}

To obtain the curve of bond - slip between the concrete and the textile used (GFG),a study of the behaviour of the interface was undertaken using the simplified finite element model of Lu et al. (2005),The different parameters taken into consideration in our case are the summarised in Table 1:

Table.1. Values of parameters characterizing the concrete interface - GFG grids

\begin{tabular}{|l|l|l|l|l|l|l|l|}
\hline Parameters & $\mathrm{f}_{\mathrm{c}}(\mathrm{Mpa})$ & $\mathrm{f}_{\mathrm{t}}(\mathrm{Mpa})$ & $\alpha$ & $ß \mathrm{~W}$ & $\mathrm{u}_{0}(\mathrm{~mm})$ & $\mathrm{G}_{\mathrm{f}}(\mathrm{Mpa})$ & $\boldsymbol{\tau}_{\max }(\boldsymbol{M} \boldsymbol{p a})$ \\
\hline \hline Values & 34.8 & 2.69 & 1.5 & 1 & 0.052 & 0,505 & 4.035 \\
\hline
\end{tabular}

After having programmed the different equations of the model and taking into account the real parameters characterizing the concrete-grids matrix interface, one obtains the curves illustrated by figures 9 and 10.

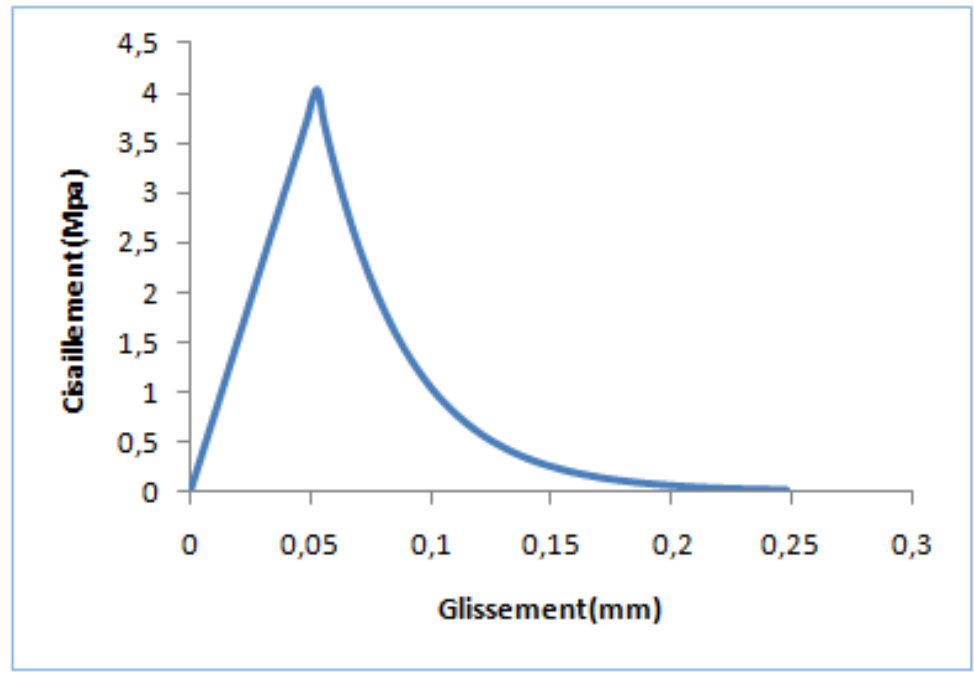

Fig.9. Bond - slip curve of the concrete-GFG interface

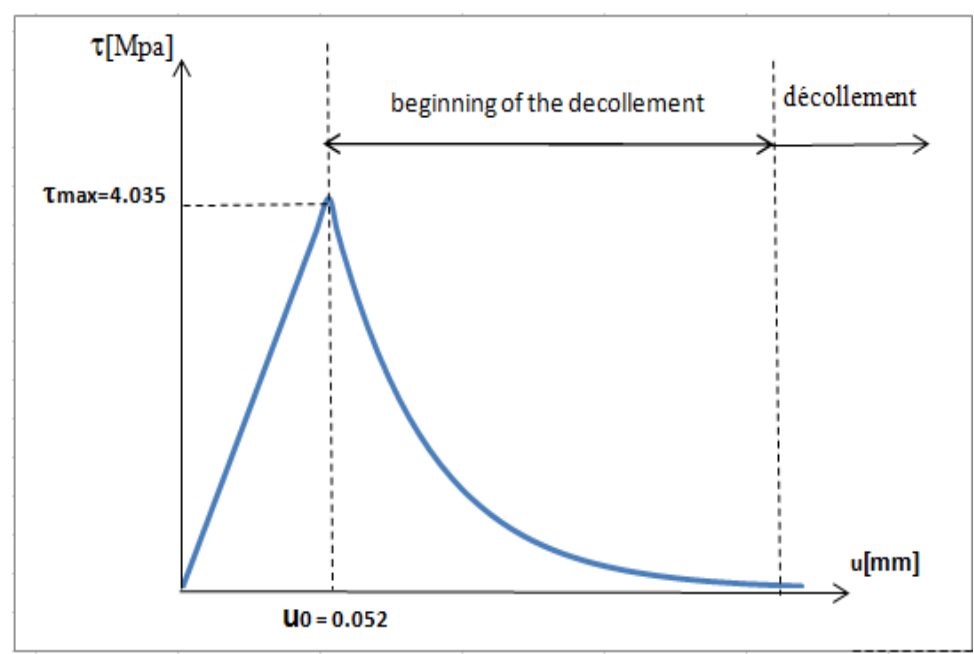

Fig.10. Illustration of the different domains of the bond - slip curve of the concrete-GFG interface 


\section{Results analysis}

From this curve, the following observations can be made:

- $\quad$ The shape of the curve is very close to the curves shown in Figures 7 and 8.

- The bond-slip curve is composed of:

$>$ A rising branch corresponding to the elastic phase, for which a significant increase in the adhesion stress is observed, whereas the slip is not significant;

$>$ A descending branch, corresponding to the beginning of the rupture of the interface, for which it is observed that the adhesion stress tends to zero when the sliding is sufficiently large.

- The initial stiffness of the bond-slip curve is much greater than the secant stiffness at the point of maximum stress. This initial high rigidity, representing the rigidity of the completely linear elastic state of the interface, decreases rapidly with the appearance of microcracks in the concrete during the increase of the adhesion stress,

- Finally, compared to the case of reinforcement by adhesive bonding of FRP composite plates or, generally, the maximum adhesion stress $\tau$ max of the order of $3 \mathrm{Mpa}$, in our case $\tau_{\max }=4,035 \mathrm{Mpa}$, which means that the adhesion of the GFG is greater than that of the FRP with the concrete

\section{Conclusions}

The experimental results highlight the advantage of reinforcing the slabs with GFG and PPG grids. Indeed, the GFG grid placed at the level of the stretched fiber increases considerably the ultimate load of the slabs subjected to the punching load, and confers a better distribution of the cracks. While the PPG grid allows the development of flexural mechanisms in the slab, which leads to a more ductile mode of rupture compared to the reference slabs.

Reinforcement with small mesh grids gives better results, because the increase in the number of roving per grid implies an increase in the percentage of the reinforcement in the concrete.

The analysis of the curve of bond-slip obtained allowed us to observe that the use of GFG and PPG grids inside the matrix concrete leads to a better behaviour of the interface between the grid and the matrix concrete, the separation of the concrete-grid interface occurs only after numerous cracks in the concrete are encountered. Nevertheless, a typical modelling of the gridconcrete interface should be established in order to have a more exact curve of bond-slip curve specific to this reinforcement case.

These conclusions highlight the value of reinforcing the slabs with GFG and PPG grids. Indeed, the GFG grid increases considerably the ultimate load of the slabs subjected to the punching and confers a better distribution of the cracks.

\section{References}

Ait Tahar, K., \& Bouzeboudja, F. (2011). Dalles en béton renforcées par des grilles composite. Matériaux \& Techniques, 99, 281-289.

Carozzi, F. G., Colombi, P., Fava, G., \& Poggi, C. (2016). A cohesive interface crack model for the matrixtextile debonding in FRCM composites. Composite Structures, 143, 230-241.

Häußler-Combe, U., \& Hartig, J. (2007). Bond and failure mechanisms of textile reinforced concrete (TRC) under uniaxial tensile loading. Cement and concrete composites, 29(4), 279-289. 
Lorenz, E., \& Ortlepp, R. (2012). Bond behavior of textile reinforcements-development of a pull-out test and modeling of the respective bond versus slip relation. In High Performance Fiber Reinforced Cement Composites 6 (pp. 479-486). Springer, Dordrecht.

Lu, X. Z., Teng, J. G., Ye, L. P., \& Jiang, J. J. (2005). Bond-slip models for FRP sheets/plates bonded to concrete. Engineering structures, 27(6), 920-937.

Soranakom, C., \& Mobasher, B. (2009). Geometrical and mechanical aspects of fabric bonding and pullout in cement composites. Materials and Structures, 42(6), 765-777.

Neale, K. W., Ebead, U. A., Abdel Baky, H. M., Elsayed, W. E., \& Godat, A. (2005). Modelling of debonding phenomena in FRP-strengthened concrete beams and slabs. Proceedings of the International Symposium on Bond Behaviour of FRP in Structures (BBFS 2005). International Institute for FRP in Construction, University of Sherbrooke. Quebec, Canada J1K 2R1.

Neale, K. W., Godat, A., Abdel Baky, H., Elsayed, W. E., \& Ebead, U. A. (2011). Approaches for finite element simulations of FRP-strengthened concrete beams and slabs. Architecture Civil Engineering Environment, 4(4), 59-72.

Wang, X., \& Wu, Z. (2010). Integrated high-performance thousand-metre scale cable-stayed bridge with hybrid FRP cables. Composites Part B: Engineering, 41(2), 166-175. 